\title{
On the Painleve property of a hydrodynamic system
}

\author{
Dmitry Noshchenko ${ }^{1,2, \star}$ and Andrey Perezhogin ${ }^{1,2, \star \star}$ \\ ${ }^{1}$ Institute of Cosmophysical Researches and Radio Wave Propagation FEB RAS, \\ 684034, Kamchatsky Kray, Paratunka, Mirnaya st., 7, Russia \\ ${ }^{2}$ Vitus Bering Kamchatka State University, \\ 683031, Petropavlovsk-Kamchatsky, Pogranichnaya st., 4, Russia
}

\begin{abstract}
We represent conditions of hydrodynamic system when it passes the Painleve test. We use Kovaleskaya-Gambie method for fourth order ordinary differential system. We obtain Lorenz-like dynamic for this system.
\end{abstract}

\section{Introduction}

The existence of magnetic fields of planets, stars and galaxy is explained by a dynamo-mechanism [1]. Mathematical aspects of dynamo effect are reduced to solutions of MHD equations. Nonlinear terms of these equations don't allow to find an analytical solutions (except private cases). Direct number simulation of 3D magnetohydrodynamics with big Reynolds number require huge computing at supercomputers [2]. So simplified MHD systems are very important to investigate for understanding main features of dynamo.

In the paper we discuss the analytical properties of a dynamical system, which is the simplest model of the dynamo. We find relation between coefficients of system in order to pass the Painleve test.

We discuss a model of process which generates the average magnetic field by a turbulence flow of viscous incompressible fluid with the alpha effect in rotating coordinate system. It is described by magnetohydrodynamics equations.

$$
\begin{aligned}
& \partial_{t} \mathbf{v}+\mathrm{R}_{\mathrm{m}}(\mathbf{v} \nabla) \mathbf{v}=\operatorname{Pm} \Delta \mathbf{v}-\nabla p-\mathrm{E}^{-1} \operatorname{Pm}\left(\mathbf{e}_{z} \times \mathbf{v}\right)+\operatorname{rot} \mathbf{B} \times \mathbf{B}, \\
& \partial_{t} \mathbf{B}=\mathrm{R}_{\mathrm{m}} \operatorname{rot}(\mathbf{v} \times \mathbf{B})+\mathrm{R}_{\alpha} \operatorname{rot}(\alpha \mathbf{B})+\Delta \mathbf{B}, \\
& \nabla \cdot \mathbf{v}=0, \\
& \nabla \cdot \mathbf{B}=0,
\end{aligned}
$$

where $\mathbf{v}$ - the average velocity, $\mathbf{B}$ - the average magnetic field, $p$ - pressure, $\mathbf{f}$ - mass density of external forces, $\alpha$ - tensor of the $\alpha$-effect, $\mathrm{R}_{\mathrm{m}}$ - magnetic Reynolds number, $\mathrm{E}$ - Ekman number, Pm - Magnetic Prandtl number, $\mathrm{R}_{\alpha}$ - amplitude of the $\alpha$-effect, $\mathbf{e}_{z}$ - the unit vector of axis of rotation.

\footnotetext{
^e-mail: d951039@gmail.com

$\star \star$ e-mail: d72156@gmail.com
} 
We admit that field axially symmetric with respect to axis $\mathbf{e}_{z}$. Solenoidal fields $\mathbf{v}$ and $\mathbf{B}$ give the sum of the toroidal and poloidal components.

We represent the following decomposition of the velocity and magnetic field into the sum of timedependent amplitudes and stationary poloidal (toroidal) fields products:

$$
\begin{aligned}
& \mathbf{v}=x_{1}(t) \mathbf{v}^{T}(\mathbf{r})+x_{2}(t) \mathbf{v}^{P}(\mathbf{r}), \\
& \mathbf{B}=y_{1}(t) \mathbf{B}^{T}(\mathbf{r})+y_{2}(t) \mathbf{B}^{P}(\mathbf{r}) .
\end{aligned}
$$

Substitution of decomposition (2) in (1) gives system of amplitude's equations [3]:

$$
\begin{aligned}
& \frac{d x_{1}}{d t}=\mathrm{R}_{\mathrm{m}} A_{112} x_{1} x_{2}+\mathrm{E}^{-1} \mathrm{Pm} P_{12} x_{2}+F_{1}+\mathrm{R}_{\mathrm{m}} L_{112} y_{1} y_{2}-\mu_{1} x_{1}, \\
& \frac{d x_{2}}{d t}=\mathrm{R}_{\mathrm{m}} A_{211} x_{1}^{2}+\mathrm{E}^{-1} \mathrm{Pm} P_{21} x_{1}+F_{2}+\mathrm{R}_{\mathrm{m}} L_{211} y_{1}^{2}+\mathrm{R}_{\mathrm{m}} L_{222} y_{2}^{2}-\mu_{2} x_{2}, \\
& \frac{d y_{1}}{d t}=\mathrm{R}_{\mathrm{m}} W_{112} x_{1} y_{2}+\mathrm{R}_{\mathrm{m}} W_{121} x_{2} y_{1}+\mathrm{R}_{\alpha} W_{1 \alpha 2} y_{2}-\eta_{1} y_{1}, \\
& \frac{d y_{2}}{d t}=\mathrm{R}_{\mathrm{m}} W_{222} x_{2} y_{2}+\mathrm{R}_{\alpha} W_{2 \alpha 1} y_{1}-\eta_{2} y_{2} \text {. }
\end{aligned}
$$

In the system we take into account that in the axially symmetric case the vector lines of any poloidal field lie in planes passing through the axis of rotation, and the lines of any toroidal field perpendicular to them. Uppercase letters denote constant coefficients. They appear after application of Galerkin's method to a system. Coefficients $\mu_{i}$ and $\eta_{i}$ determine the dissipation rate of velocity and magnetic fields modes from (2). In this case we assume $P_{12}=-P_{21}$ and some other relations on coefficients $A_{112}=-A_{211}, L_{112}=-W_{112}, L_{211}=-W_{121}, L_{222}=-W_{222}, \mu_{1}=\mu_{2}, \eta_{1}=\eta_{2}, F_{1}=0$.

System (3) is the simplest dynamo model without a kinematic effect. In this paper we investigate its analitical properties.

\section{Simplified ODE system}

We present the Painleve test on simplified ODE system. The Painleve test is neccesary condition for the Painleve property. The formal solution is given by Laurent series near a movable singularity $x-x_{0}$. For this aim we use Kowalevski-Gambier method [4].

Main steps of Kowalevski-Gambier method are

1. substitution $u(x)=u_{0} x^{p}$ in order to find integer value of parameter $p$.

2. computation coefficients $u(x)=u_{j} x^{p+j}$ for integer $j$. For every fixed $j$ we have a linear algebraic system on coefficient $u_{j}$ of Laurent series. If a system is consistent we have an uniformal solution for fixed $j$. For some values of $j$ system can be overdefined. In this case coefficients of Laurent series are free. In order to determine $j$ for which system is overdetermined we can calculate Fuchs indices.

3. If a linear algebra system of Laurent series coefficients for each Fuchs indices is consistent the ODE system passes Painleve test. It does not imply the Painleve property. If system is inconsistent ODE system passes test. 
Let us discuss one simplified case of (3). We suppose that $L_{222}=L_{211}=W_{121}=W_{222}=F_{1}=0$, $F_{2}=M, R_{\alpha} W_{2 \alpha 1}=R_{\alpha} W_{1 \alpha 2}=\alpha, P_{12}=K, P_{21}=-K, x_{1}=u_{1}, x_{1}=u_{2}, y_{1}=u_{3}, y_{2}=u_{4}$, independent variable $t=x$. So a reduced ODE system is

$$
\left\{\begin{array}{l}
\dot{u_{1}}=-\lambda u_{1}+K u_{2}-L u_{3} u_{4} \\
\dot{u_{2}}=-\lambda u_{2}-K u_{1}+M \\
\dot{u_{3}}=L u_{1} u_{4}+\alpha u_{4}-u_{3} \\
\dot{u_{4}}=\alpha u_{3}-u_{4}
\end{array}\right.
$$

where $M, L, K, \lambda, \alpha$-independent parameters.

1 step. We substitute $u_{i}(x)=u_{i, 0} x^{p_{i}}, i=1 . .4$ at system (4). Main terms give next system on $p_{i}$ : $p_{1}-1=p_{3}+p_{4}, p_{2}-1=p_{1}, p_{3}-1=p_{1}+p_{4}, p_{4}-1=p_{3}$. Solution of the linear system is $p_{1}=-2, p_{2}=-1, p_{3}=-2, p_{4}=-1$.

We find coefficients $u_{i, 0}$ from system of main terms for calculated values of $p_{i}, i=1 . .4$ :

$p_{1} x_{1}=-L x_{3} x_{4}, p_{2} x_{2}=-K x_{1}, p_{3} x_{3}=L x_{1} x_{4}, p_{4} x_{4}=\alpha x_{3}$

From this system we get one trivial solution and four nontrivial

$$
u_{1,0}=\frac{2}{L \alpha}, u_{2,0}=\frac{2 K}{L \alpha}, u_{3,0}= \pm \frac{2 I}{L \alpha}, u_{4,0}= \pm \frac{2 I}{L},
$$

where $I$ - the imagery unit.

Let us check the first solution $u_{3,0}=\frac{2 I}{L \alpha}, u_{4,0}=\frac{2 I}{L}$, when $u_{3,0}, u_{4,0}$ have the same sign. In this case Fuchs indices are irrational $0,1,5 / 2-1 / 2 \sqrt{17}, 5 / 2+1 / 2 \sqrt{17}$. Because of the irrational value we need to take $u_{3,0}, u_{4,0}$ with the different sign. For positive values of $u_{3,0}$ and $u_{4,0}$ the Fuchs indices are $-1,1,2,4$. For each Fuchs index we get 3 invariants:

$$
\begin{gathered}
Q_{1}=\frac{8(\lambda-1)}{\alpha L}, Q_{2}=-2 \frac{K^{2}+\lambda^{2}+4 \lambda-4}{L \alpha} \\
Q_{4}=\left((\lambda-2) u_{2,1}-u_{2,2}\right) K+4 \frac{(\lambda-1)\left(\alpha^{2} \lambda-\lambda^{3}-2 \lambda+2\right)}{L \alpha}+\frac{\lambda(3 \lambda-2)\left(-u_{2,1} \lambda+M-u_{2,2}\right)}{K}
\end{gathered}
$$

Assuming $Q_{1}=Q_{2}=Q_{4}=0$ we get next set of system's parameters $\lambda=1, K= \pm I, M=0$.

\section{General ODE system}

In this case we assume $P_{12}=-P_{21}$ and some other relations on coefficients $A_{112}=-A_{211}=A$, $L_{112}=-W_{112}=-L, L_{211}=-W_{121}=-P, L_{222}=-W_{222}=-Q, \mu_{1}=\mu_{2}=\eta_{1}=\eta_{2}=1, F_{1}=0$. For such coefficients system is more general than previos one.

$$
\begin{aligned}
& \dot{u_{1}}=A u_{1} u_{2}+K u_{2}-L u_{3} u_{4}-u_{1} \\
& \dot{u_{2}}=-A u_{1}^{2}-K u_{1}-P u_{3}^{2}-Q u_{4}^{2}+M-u_{2} \\
& \dot{u_{3}}=L u_{1} u_{4}+P u_{2} u_{3}+\alpha u_{4}-u_{3} \\
& \dot{u_{4}}=Q u_{2} u_{3}+\alpha u_{3}-u_{4}
\end{aligned}
$$

Let us consider the Painleve test of system (5) with $A=P$. First we check if the pivot terms satisfy following equations

$$
\begin{aligned}
& p_{1}-1=p_{1}+p_{2}, p_{1}-1=p_{3}+p_{4}, p_{2}-1=2 p_{1}, p_{2}-1=2 p_{3}, p_{2}-1=2 p_{4}, \\
& p_{3}-1=p_{1}+p_{4}, p_{3}-1=p_{2}+p_{3}, p_{4}-1=p_{2}+p_{3}
\end{aligned}
$$



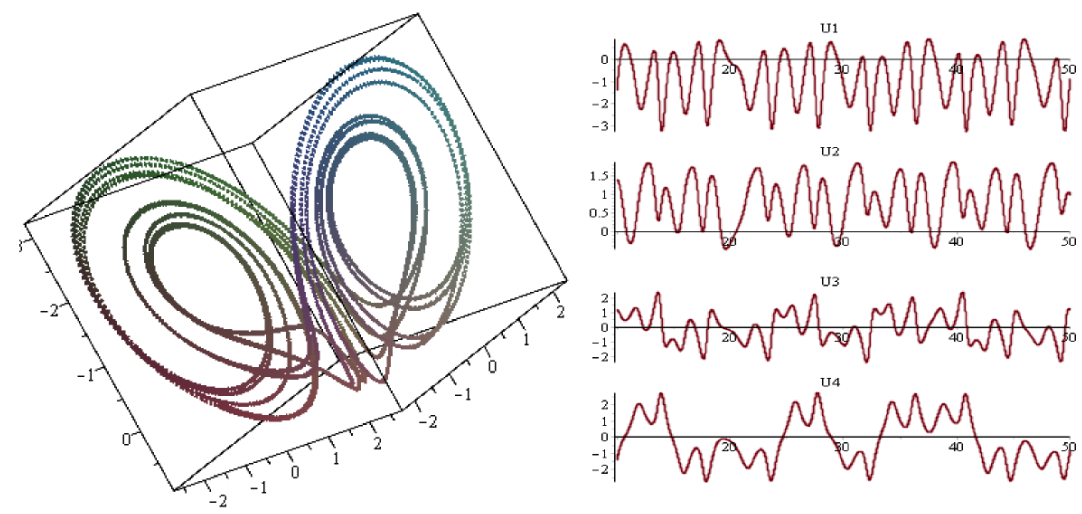

Figure 1. One velocity and two magnetic cmponents

There is one case when $p_{1}=-1, p_{2}=-1, p_{3}=-1, p_{4}=-1$. Now we substitute expantions $u_{i}=u_{0, i} x^{p_{i}}$ into pivot terms and get nonlinear system for coefficients $u_{0, i}$.

$$
u_{0,1}=I u_{0,3}, u_{0,2}=\left(L Q u_{0,1}-P\right)^{-1}, u_{0,3}=\frac{I L+\sqrt{-L^{2}-4 P Q}}{2 Q^{2}}, u_{0,4}=-Q u_{0,2} u_{0,3}
$$

In order to simplify $u_{0, i}$ we put $Q=-L^{2} /(4 P)$. Such simplification gives a very simple characteristic equation for the Fuchs indices $j\left(j^{3}-6 j^{2}+5 j+12\right)$. Solving equation we obtain indicies $-1,0,3,4$. A characteristic polynomial doesn't depend on parameters $L, P$. There are two invariants for this system. Invariants correspond to the Fuchs values $j=3$ and $j=4$. Detailed analysis of the invariants gives some relations on coefficients. So $L=2 P, P=1$. Free parameters are $K, \alpha$ and dependent one is $M$. Resulting system passes the Painleve test with $\alpha=3, K=2$.

$$
\begin{aligned}
& \dot{u_{1}}=u_{1} u_{2}-u_{1}+2 u_{2}-2 u_{3} u_{4}, \\
& \dot{u_{2}}=-u_{1}^{2}-u_{2}-2 u_{1}-u_{3}^{2}+u_{4}^{2}+\frac{1330}{99}, \\
& \dot{u_{3}}=2 u_{1} u_{4}+u_{2} u_{3}+3 u_{4}-u_{3}, \\
& \dot{u_{4}}=-u_{2} u_{3}+3 u_{3}-u_{4}
\end{aligned}
$$

If we put value of $M$ to zero, we get a modification of (6). Direct solutions of this system gives periodic oscillations (fig. 1). If we keep constant force $M$, the periodic solutions will be relaxation oscillations.

\section{Conclusion}

We have considered two cases of hydrodynamic system. For these systems we find sets of parameters to satisfy the Painleve test. It is possible to check what conservation law holds for this system.

\section{References}

[1] Zel'dovich B., Ruzmaikin A.A., Sokolov D.D. Magnetic fields in astrophysics. - MoscowIzhevsk: RHD, 2006. - 384 p. In Russian.

[2] Frick P.G. Turbulence: approaches and models. - Moscow-Izhevsk: RHD, 2010. - 332 p. In Russian. 
[3] Gledzer E.B., Dolzhansky F.V., Obukhov A.M. Systems of hydrodynamic type and their application. - M .: Nauka. 2010. In Russian.

[4] Conte R.M., Musette M. The Painlevé Handbook. Springer Netherlands. 2008. 